\title{
ROLE OF ANTI-INFLAMMATORY DRUGS IN TRAUMATIC ARDS
}

Siddani Srinivas ${ }^{1}$, Rakesh Chintalapudi², Pudi Rama Rao ${ }^{3}$

\section{HOW TO CITE THIS ARTICLE:}

Siddani Srinivas, Rakesh Chintalapudi, Pudi Rama Rao. "Role of Anti-Inflammatory Drugs in Traumatic ARDS". Journal of Evolution of Medical and Dental Sciences 2014; Vol. 3, Issue 43, September 11; Page: 10679-10682, DOI: $10.14260 /$ jemds/2014/3391

ABSTRACT: ALI/ARDS is an acute pathological process with dynamic and complex inflammatory response. In traumatic ARDS inflammatory response plays major role in outcomes. Antiinflammatory agents may play major role in recovery. We report successful management of early traumatic ARDS with lung protective strategy along with Ant-inflammatory agents. Larger trails are needed to assess Ant-inflammatory agents in ALI/ARDS.

KEYWORDS: ALI/ARDS, Inflammatory Response, Steroids, Ulinastatin.

INTRODUCTION: ARDS is a dynamic and complex inflammatory response. ARDS in trauma patients, though identified in 1967, by Ashbaugh, and Levine², Sepsis ARDS, is more evaluated than trauma ARDS in many studies. The trauma population constitutes a more homogeneous ARDS group with greater physiological reserve because of their lower age and lesser co-morbidity. Recent analysis of National Trauma Data Bank shows, the incidence of Trauma related ARDS to be $6.5 \%,{ }^{1}$ requiring Ventilation for more than 48 hours. Two forms of ARDS have been described 1) Early ARDS: during first 48 hours after Trauma and related to Haemorrhagic Shock 2) Late ARDS: after 48 hours and more related to Organ Failure and Sepsis. Here we present early ARDS without Haemorrhagic Shock.

CASE REPORT: A male patient of 31 year old with no co morbidity, sustained crush injury with both bone fracture of right leg when a stationary vehicle rolled on its own. There are no other external injuries seen. Patient GCS in ER in 15/15 with stable hemodynamics' and maintaining saturation on room air His routine investigation including chest $x$-ray are within normal limits. Immediate fixation of bones was not considered as patient wants to get surgery done through government sponsored programme.

Patient was managed symptomatically and shifted to floors. After 3 hours patient started complain of SOB and tachypnea his saturation came down to $84 \%$ and with oxygen supplementation with 10 liters, Sp02improved to $90 \%$ patient but, patient continue to have tachycardia and tachyopnea with normal blood pressure. ABG and Chest x-ray, ECG along with CBC, CRP, and D-dimer immediately sent. His ABG showed PO2 48 and pCO2 36 pH 7.416 Hco3 is 23, chest x-ray showed bilateral infiltrates, his ECG is normal, CRP level positive $-11.0 \mathrm{mg} / \mathrm{dl}$ with no drop in $\mathrm{Hb} \%$ and Ddimer negative.

A provisional diagnosis of Fat Embolism Syndrome / ARDS was considered. Patient was kept on NIV S/T with 15 liter of Oxygen and IPAP 14 EPAP 10, after one hour his ABG was Po2 50.7 Pco2 $38.3 \mathrm{pH} 7.24$ with respiration rate more than 35 per minute. Patient was intubated and connected to ventilator, he is ventilated in pressure control mode with lung protective strategy and PEEP of 12, his CBC did not show thrombocytopenia no rash was observed, his 2D Echo was normal. His fat embolism score is low. He is ventilated on first day with fentanyl infusion 50-100 mic per hour and vecuronium 4 to $8 \mathrm{mgs}$, whenever needed. 
Patient was kept on Inj. Ceftriaxone one gm bid, Injection Hydrocortisone 100 milligram bolus followed by 10 milligram/hr infusion, injection Ulinastatin one lac units Tid, and LMWH 0.4 SC OD. His 2nd day ABG PO2 121 PCO2 19.4 and HCo3 25.6 with FiO2 0.6 and PEEP 12, his "crp" level is positive $6.1 \mathrm{mic} / \mathrm{dl}$ hydrocortisone infusion continued.

On $3^{\text {rd }}$ day his ABG showed Po2 85.7 with Fio2 0.4 and PEEP 8,crp level is 4.8mg/dl he is maintained with light sedation and spontaneous breath trail given, injection hydro cortisone to converted to 100 milligram to TID, bronchoscopy was done, which is with in normal limit and BAL showed no growth and fat globules. On 5the day patient was converted to spontaneous mode with PEEP six and FiO2 .35,his ABG showed p02 106 and he is Extubated and shifted to floor after one day, his CRP level is 3.4 micro grams / dl on day 5.

DISCUSSION: Trauma has been identified to constitute 7\% of ARDS cases the incidence of ARDS did not differ between blunt and penetrating mechanism of injury. The incidence of ARDS in trauma was higher in older age group and female population. ${ }^{9}$ The mortality of trauma - ALI is estimated at $24 \%$. The 60 day mortality day rate of trauma patient in ARDS network trail hospital is a 10\%, it has not seen any further decrease in recent times.

The mortality between female and male patients with ARDS after traumatic injury does not appear to differ. The mortality of ARDS in victims of trauma is lower than those with ARDS from other causes after adjusting for baseline differences. Over the last decade ${ }^{3}$ mortality rate have continue to decrease in the general population with ALI / ARDS, this changes might be because of change in ventilation strategies, fluid management and overall organ specific management.

A 2006 retrospective review of trauma ICU data ${ }^{1}$ at the University of Southern California showed an overall complication rate of $43 \%$ in patient with ARDS. Complication included Pneumonia (50\%), deep venous thrombosis, Pulmonary Embolism, acute renal failure and DIC. ARDS patient had longer hospital stage (32days), longer ICU stays (22days), crude mortality rate is19\% and higher hospital charges.

Independent risk factors for the 1development of ARDS after trauma are those with an injury severity score (ISS) > 25, increasing Acute Physiology and Chronic Health Evaluation II (APACHE II) scores, the presence of pulmonary contusion, a large transfusion requirement, hypotension on admission, age $>65$ years, duration of mechanical ventilation along with delayed stabilization of femoral bone fracture.

ARDS following trauma is different in many respects from ARDS related to other causes. Patients with ARDS after injury tend to be younger and have fewer comorbid medical conditions. Endothelial activation factor levels are lower in patients with ARDS related to trauma as opposed to septic insults.

Following cellular injury, endothelial cells can become activated, a principal mechanism in the complex pathologic events that result in ARDS. Subgroup analysis of ARDS Network data ${ }^{4}$ has shown significantly lower levels of serum biomarkers (von Willebrand factor antigen [vWF], surfactant protein D [SP-D], tumour necrosis factor receptor-1 [TNFr-1], and intercellular adhesion molecule-1 [IC A M-1], associated with poor outcome in trauma patients.

This patient had crush injury with long bone fracture and no other injury he had stable vitals and conscious and coherent. He developed symptoms within 4hours from inciting injury and developed hypoxia and bilateral infiltrates on chest X-ray, his 2D Echo is normal. 
Differential diagnosis of FES considered but except for hypoxia, tachycardia and tachyopnea no other criteria met (Gurd and Wilson criteria). Lung contusion and pulmonary haemorrhages are considered but no feature of chest trauma seen. Patient didn't receive any blood transfusion or massive fluid resuscitation.

Patient was managed with lung protective strategy, conservative fluid management. His CRP a nonspecific marker of inflammation is high, so patient is kept on hydrocortisone infusion andUlinastatin,7,8 a serine protease inhibitor which inhibits several pro-inflammatory proteases and decreases inflammatory cytokine levels, to decrease the inflammatory changes. It is observed that the CRP levels decreased have the patient conditions improved. Therapeutic usage of Ant-inflammatory agents might help in decreasing complications.

The role of steroids ${ }^{5,6}$ in traumatic ARDS, not much evidence is available, but the recent metaanalysis showed that early steroids treatment in ARDS decreased ventilator days, ICU days, and hospital stay. The use of Ulinastatin is initiated with hope of decreasing SIRS response. The role of Ant-inflammatory needs consideration in early ARDS. Larger studies and controlled studies regarding efficacy of steroid with or without Ulinastatin is required.

\section{REFERENCES:}

1. Acute lung injury and the acute respiratory distress syndrome in the injured patient Bakowitz et al. Scandinavian Journal of Trauma, Resuscitation and Emergency Medicine 2012, 20: 54.

2. Ash baugh DG, Bigelow DB, Petty TL, Levine BE: Acute respiratory distress in adults. Lancet 1967, 2 (7511): 319-323.

3. Zambon M, Vincent JL: Mortality rates for patients with acute lung injury/ARDS have decreased over time. Chest 2008, 133 (5): 1120- 1127.

4. Early markers of acute respiratory distress syndrome development in severe trauma patients Journal of Critical Care (2006) 21, 253 - 258.

5. Pharmacological interventions in acute respiratory distress syndrome Roch et al. Annals of Intensive Care 2013, 3: 20.

6. Corticosteroids in the prevention and treatment of Acute Respiratory Distress Syndrome (ARDS) in adults: meta-analysis John Victor Peter, Physician.

7. The anti-inflammatory effects of Ulinastatin in patients with Traumatic Haemorrhagic ShockPark KH. J Med Science, 2010 Jan 25(1): 28-34.

8. Effect of Ulinastatin in traumatic brain injury with multiple injuries Tu Y etal 2012, Nov 24 (11): 677-9.

9. Effect of age on the development of ARDS in trauma patients. Craig J. Johnston, et al. 2002 American College of Chest Physicians Conference. 


\section{CASE REPORT}

\section{AUTHORS:}

1. Siddani Srinivas

2. Rakesh Chintalapudi

3. Pudi Rama Rao

\section{PARTICULARS OF CONTRIBUTORS:}

1. Chief Intensivist \& Medical Director, Department of Anaesthesia \& critical Care, Manipal Hospital, Maharanipeta, Visakhapatnam.

2. Assistant Professor, Department of Anaesthesia, Andhra Medical College, King George Hospital, Visakhapatnam.

3. Intensivist, Department of Anaesthesia \& critical Care Manipal Hospital, Maharanipeta, Visakhapatnam.

\section{NAME ADDRESS EMAIL ID OF THE CORRESPONDING AUTHOR:}

Dr. Rakesh Chintalapudi,

Bay Drive Prince Apartments,

F. No: A-1 Ground floor,

Nowroji road, Maharanipeta,

Visakhapatnam-530002,

Andhra Pradesh.

Email: rakesh1959@gmail.com

Date of Submission: 27/08/2014.

Date of Peer Review: 28/08/2014.

Date of Acceptance: 05/09/2014.

Date of Publishing: 10/09/2014. 